\title{
ENTRE EL HEDONISMO, LA ESPECULACIÓN Y LA NECESIDAD El caso del Parque Pignatelli de Zaragoza
}

\author{
Laura Ruiz Cantera \\ Universidad: Universidad de Zaragoza y miembro del grupo de investigación consolidado OAAEP, \\ financiado por el Gobierno de Aragón con fondos del FSE. \\ Director de la tesis: Dra. Isabel Yeste Navarro \\ Email: Irucan@unizar.es
}

\section{RESUMEN}

El objetivo principal de la presente investigación es mostrar y reflexionar sobre los intereses que se entrecruzaron en la creación del parque Pignatelli, primer parque construido en Zaragoza, cuyo proyecto data del año 1892. En este sentido, se puede apuntar que este espacio verde nació bajo aspiraciones y concepciones burguesas que conjuraron intereses económicos sobre la zona, a ello sumar la necesidad de incorporar un lugar de ocio y esparcimiento ciudadano y también, de crear una zona bucólica a emulación de otras ciudades.

Una interdisciplinariedad que exige abarcar un mayor número de líneas de investigación, siempre apoyadas en la consulta de fuentes documentales. De ahí, que para dar sentido de una forma coherente y completa a este trabajo se ha precisado el estudio de un amplio compendio documental y cartográfico procedente del Archivo y Hemeroteca Municipal de Zaragoza.

Palabras clave: Urbanismo, Zaragoza, parque urbano, parque Pignatelli, espacios verdes

\section{ABSTRACT}

The main objective of the present research is to show and reflect on the interests that were intertwined in the creation of the park Pignatelli, the first park built in Zaragoza, whose project dates from 1892. In this sense, it can be pointed out that this green space was born under aspirations and bourgeois conceptions that conjured economic interests over the area, adding to the need to incorporate a leisure place and citizenship and also to create a bucolic area to emulate other cities.

Is an interdisciplinary study that requires to cover a greater number of lines of research, always supported by the consultation of documentary sources. Hence, in order to give a coherent and complete sense to this work, it has been necessary to study an extensive documentary and cartographic compendium from the Archive and Municipal Library of Zaragoza.

Key words: urban planning, Zaragoza, urban park, Pignatelli park, green areas 


\section{CONCEPCIONES SOBRE EL PAISAJE URBANO VERDE DECIMONÓNICO EN LA CIUDAD DE ZARAGOZA: EL CAMINO HACIA LA SENSIBILIZACIÓN}

\section{1. Introducción}

Durante el siglo XIX, la Revolución Industrial dio lugar a cambios significativos que afectaron en la manera de vivir del ser humano. La localización de las industrias y la integración de los tendidos ferroviarios en el interior de la ciudad y el éxodo de los trabajadores del campo produjeron contaminación, vertido de desechos, intrusiones visuales y superpoblación. Inglaterra y Alemania fueron los primeros países en sufrir las consecuencias de la industrialización y desde estos puntos geográficos se alzaron las primeras voces que postularon en contra de la ciudad industrial. A partir de aquí se inició una búsqueda para sanar lo síntomas de una ciudad enferma mediante la modificación del trazado urbano con la integración de espacios que ofrecían visiones de una naturaleza domesticada. El verde urbano se convirtió en una píldora espiritual, sanadora, ideológica y social.

Los primeros parques públicos se desarrollaron en países como Inglaterra, donde se produjo un salto del jardín señorial al parque urbano y de lo privado a lo público con fines especulativos.

En España, la incorporación de los parques urbanos en las ciudades fue desigual debido a dos motivos principales: la incidencia de los efectos de la industrialización no fue homogénea en el territorio español y por lo tanto, tampoco lo fueron las transformaciones sociales producidas por el capitalismo industrial y su máximo representante, la burguesía. En consonancia con ello, los intereses de esta clase dominante por el espacio urbano y sus beneficios no se desarrollaron a la par en todas las ciudad españolas, lo que hizo que el interés por la naturaleza en el seno de los poderes locales fuera disímil y se tradujera en diferentes líneas de actuación. A todo ello hay que sumar el elevado valor del suelo urbano. Fue en Barcelona donde se llevó a cabo con mayor brillantez la gestión de los parques urbanos, constituyéndose en la primera mitad de siglo parques como el de Pedralbes, de Monjüic y de Guinardó.

Dentro de este amplio espectro de circunstancias que se entrecruzan en la aparición de los primeros parques urbanos españoles, hay que tratar de confirmar cuáles fueron los verdaderos motivos por los que el verde urbano se convirtió en objetivo de los municipios y de sus clases dominantes, así como las razones de su éxito y continuación a lo largo del tiempo.

Para ello, se ha tomado como ejemplo el primer parque construido en la ciudad de Zaragoza, el parque Pignatelli. Ahora, no se busca ahondar en su dilatada historia constructiva, sino lo que se pretende en las próximas líneas es descifrar todos los aspectos que confluyeron en su ejecución y que discurrieron desde los ideales profilácticos hasta los especulativos.

\section{2. Una aproximación a los espacios verdes de la Zaragoza decimonónica}

La Zaragoza decimonónica es evocada como una ciudad fracturada por la guerra de los Sitios (1808-1809). Su tradición ambiental basada sobre todo en caminos y terrenos arbolados fue mermada por las estrategias militares donde prácticamente se talaron todos los árboles para facilitar las operaciones bélicas, unos espacios naturales que permanecieron sin reconstruir durante muchos años.

Hasta el momento del asedio, las zonas vegetales más espaciosas se situaban en la margen izquierda del Ebro por ser una zona que se inundaba con frecuencia, como eran la Arboleda de Macanaz, las Balsas de Ebro Viejo o la extensión verde situada junto el meandro de Ranillas. Mientras que la urbanización de Zaragoza, se había desarrollado sobre la margen derecha del Ebro por ser una terraza más elevada.

En el núcleo urbanizado la presencia de la naturaleza quedaba constatada mediante las rondas, los caminos arbolados, las plazas ajardinadas y también por el Jardín Botánico, emplazado junto a la Huerta de Santa Engracia y la calle de San Miguel, construido en 1798 por la Real Sociedad Económica Aragonesa de Amigos del País con fines académicos relacionados con la botánica, la farmacéutica, etc. Éste último es un signo de la relación que existió entre la jardinería y la instrucción agrícola desde finales del siglo XVIII.

Los paseos se construyeron, en primer lugar en el límite de la ciudad, definiendo y reorganizando dicho límite. También en los caminos que partían de la ciudad; y finalmente en la misma trama urbana (Capel, 2002: 251). Entre ellos el más importante y frecuentado fue el llamado de "Santa Engracia" — junto al 
monasterio Jerónimo de Santa Engracia-, y que como describe J.J. López González (1985:215) estaba "localizado en un lugar muy frecuentado por transeúntes de todas las clases sociales, sobre todo dos días al año, con motivo de las festividades de San Juan Bautista y San Pedro, en que las paseaban bajo la sombra que proporcionaba la arboleda del paseo, eran amenizadas por la música interpretada por las bandas de los regimientos acuartelados en la ciudad". Las obras de remodelación del citado paseo comenzaron a finales del siglo XVII, aunque el verdadero impulso se acometió en las últimas décadas del siglo XVIII, momento en el que se construyeron los bancos de piedra y se pobló el paseo de árboles. Tampoco se descuidó el paseo la ribera del Ebro, y así, con motivo de las fiestas del Pilar del año 1789, se dio fin a la obra de empedrado del paseo. También a finales de siglo se produce la apertura del paseo de Torrero con motivo de la inauguración del Canal Imperial, convirtiéndose en eje del ensanche hacia el Sur de la ciudad.

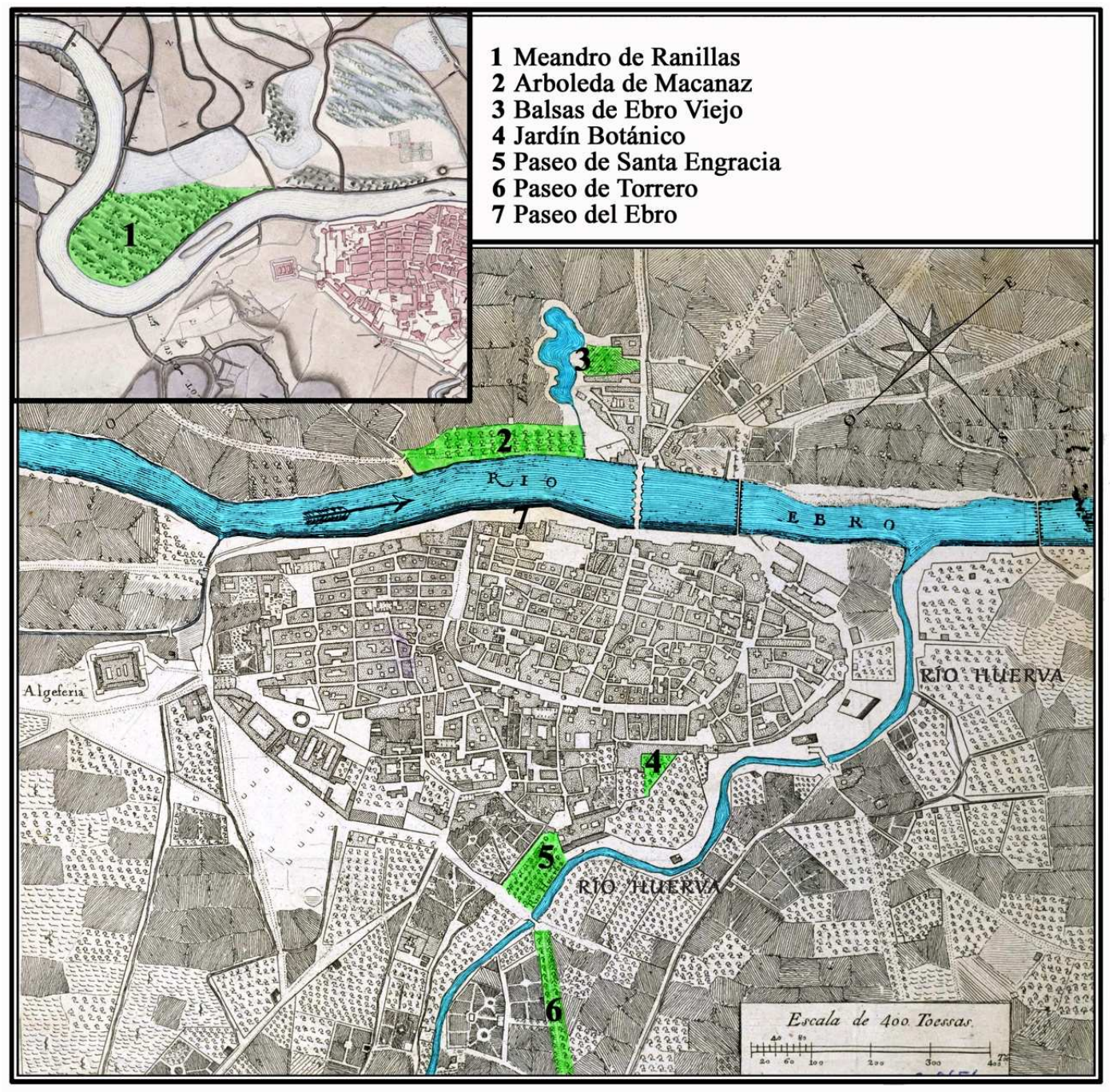

Plano topográfico de la ciudad de Zaragoza en 1778 (anónimo). Fuente: www.adioszaragoza.blogspot.com

Un impulso para la introducción de las zonas verdes en la ciudad fue originada por el establecimiento de las tropas francesas durante los Sitios. El asedio francés en la capital (1809-1813) supuso un intento de trasladar las teorías urbanísticas sobre el embellecimiento e higiene francesas, como la integración de parques y jardines, el trazado urbano regular y ordenado, el empedrado y la alineación de calles; procesos de transformación que desde principios del siglo XVIII se habían acometido en ciudades de Francia como Nancy, Metz y París. En Zaragoza, estos criterios se hicieron factibles en el paseo Imperial — paseo de la Independencia-, proyectado por el maestro de obras Joaquín Asensio Martínez en 1811. Durante el reinado de Fernando VII, esta iniciativa fue retomada por Martín Garay en 1815, quién ordenó el espacio y concibió un paseo arbolado que finalizaba con la nueva puerta y tras ella se remató con una "glorieta" ajardinada. En la década de los treinta, Tiburcio del Caso llevó a cabo la remodelación del entorno y con 
Isabel II se planteó la terminación del paseo tomando como modelo la parisina Rue Rivoli de Percier y Fontaine bajo las directrices de los arquitectos Yarza y Gironza, que también dieron forma oval a la glorieta, destinada para jardines (Yeste, 2004).

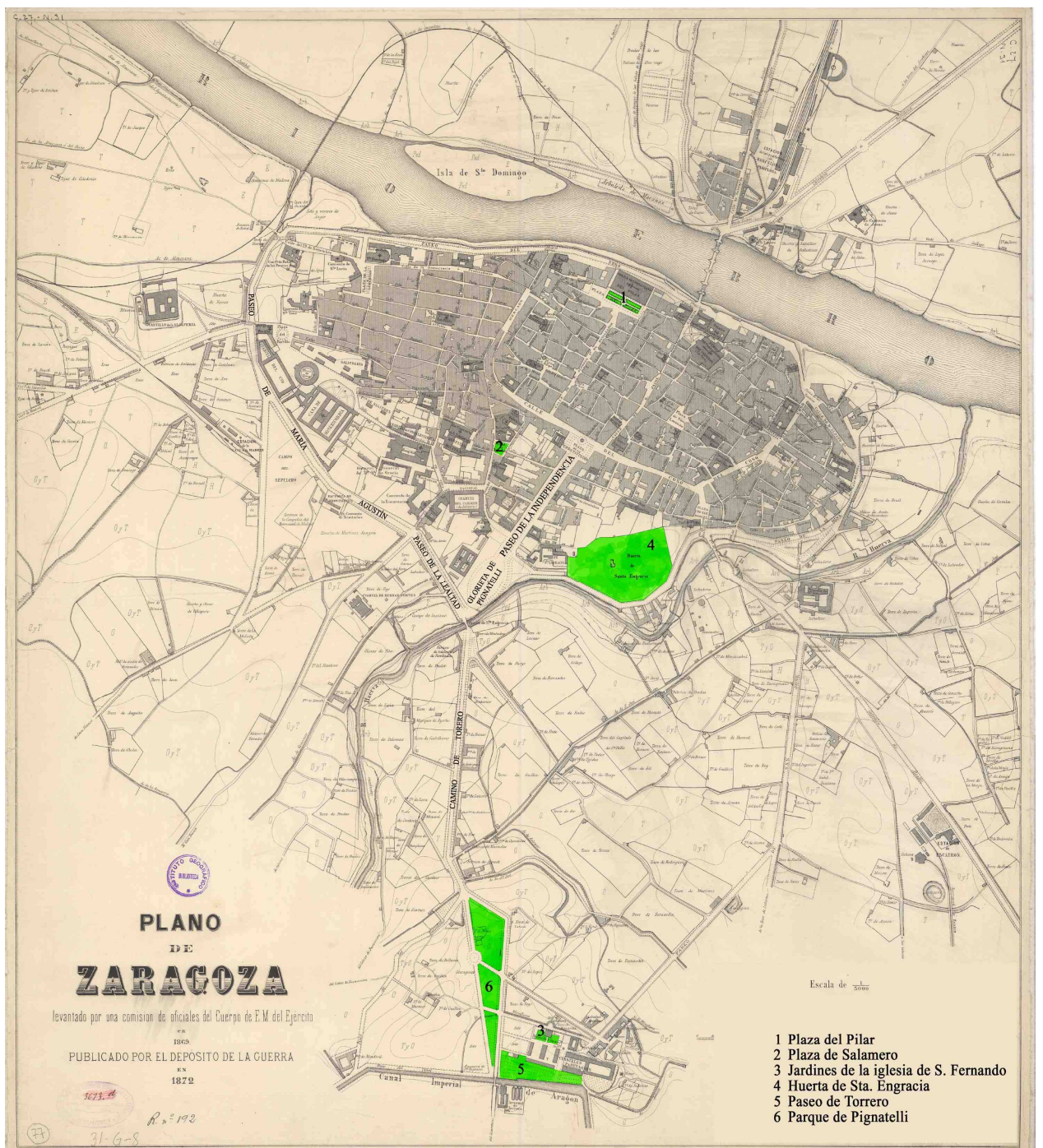

Plano de Zaragoza de 1869 (Levantado por una comisión de oficiales del Cuerpo del E.M del Ejército y publicado por el Depósito de Guerra en 1872). Fuente: http://www.ign.es/fondoscartograficos/

En la primera mitad del siglo XIX, el paseo arbolado siguió siendo el elemento más característico del escaparate verde zaragozano, como retrata Madoz (1985: 364): "Hermosos y variados paseos, y en todas direcciones circundan la c. de Augusto: casi todo su recinto ofrece la mas deliciosa y alhagüeña perspectiva por su mucho arbolado". Haciendo alusión a la situación de Zaragoza, añade (1985: 296):

"La inmensa llanura en la que se encuentra, limitada al N. y S. Por dos bajas cord.[cordilleras] que corren paralelas al Ebro, está sembrada por multitud de árboles de varias especies, de espesos arbustos y de álamos piramidales, que a manera de cipreses parece quieren conducir á un respetuoso monumento".

Gracias a la incorporación paulatina de Zaragoza a su contemporaneidad, al establecimiento de un sistema liberal y al aumento del poder burgués, el tema de los espacios verdes amplió su abanico y se convirtió en una asunto de primer orden para el municipio durante las últimas décadas del siglo. Algunos ejemplos de este creciente interés se observa en la construcción y remodelación de jardines públicos como los que adornaban la plaza del Pilar (1867 y 1883) o la plaza Salamero (1896) y los de la iglesia de San Fernando de Torrero, construidos a finales del siglo XVIII y mejorados en 1853. 
En las últimas décadas del siglo XIX la ciudad de Zaragoza creció hasta el punto de duplicar su población, a pesar de la expansión hacia el Sur en el interior todavía quedaban terrenos libres a causa de las desamortizaciones como era la Huerta de Santa Engracia, un espacio céntrico y muy adecuado para el establecimiento de una exclusiva zona residencial. Así lo entendió el arquitecto Félix Navarro cuando redactó el Ensanche Parcial de la ciudad hacia el mediodía y el Anteproyecto de parque de Zaragoza en 1880 , siendo el planteamiento más temprano relacionado con un parque urbano para la ciudad. Aunque finalmente no se ejecutó, marcó un punto y aparte en el estudio de las zonas verdes aragonesas con la introducción de una nueva tipología. Finalmente, esta idea primigenia desembocó en la construcción de un parque público a las "afueras" de la población, en el barrio de Torrero, a principios del siglo XX. Éste fue el primer parque construido en Zaragoza, el hoy en día conocido como parque Pignatelli.



Anteproyecto de parque de Zaragoza —retocado por Isabel Yeste- (Félix Navarro, 1880). Fuente: Archivo Municipal de Zaragoza.

\section{3. El camino hacia la sensibilización}

El primer paso para la creación de un espacio público acorde al bienestar ciudadano vino dado por la mejora de las condiciones higiénico-sanitarias de la población y del aspecto urbano.

El camino fue iniciado durante la dominación francesa y continuado con actuaciones puntuales al frente de la nueva administración liberal como la construcción del Cementerio de Torrero (1834), la alineación de calles, la regularización altura de las casas, la renovación del alumbramiento con faroles de reverbero, la preocupación por el empedrado de las calles, la renovación de pavimentos callejeros etc. (Forcadell, 1998: 35-38).

Todas las actuaciones comenzaban a cubrir la problemática de la salud pública y el embellecimiento urbano, por ello, en el año 1862 la Sección 5o del Ayuntamiento tomó la decisión de crear el puesto de director de Paseos, Jardines y Arboledas con el propósito de regular las construcción de estos espacios públicos de la ciudad. El encargado de estas cuestiones había sido el Guarda Mayor de la ciudad, pero ahora se precisaba una persona culta y entendida, con una capacidad y un conocimiento más específico sobre este ámbito. ${ }^{1}$ Una de las mejoras que motivó la creación de este puesto fue la necesidad de actuar en los jardines de la puerta de Santa Engracia, que como puntualizó la Sección 5o "no son otra cosa que un hacinamiento de vegetación frondosa de plantas espontáneas y producto de años anteriores", y también urgía embellecer la afueras de la población. El elegido para desempeñar el cargo fue el ingeniero agrónomo

\footnotetext{
${ }^{1}$ Archivo Municipal de Zaragoza [A.M.Z]. Caja 1.417, exp. 902/1862.
} 
zaragozano Antonio Berbegal y Celestino. Como manifiesta Berbegal en la "Memoria sobre el ramo de arbolados y jardines de Zaragoza" ${ }^{2}$ son muchas las mejoras que se estaban introduciendo en la capital bajo su encargo, como la repoblación de los arbolados de "ornato e higiene pública" y la constitución de viveros y sotos que reprodujo gráficamente complementando las descripciones. La otra cara de moneda la ejemplifica con el estado en que se encontraba la Puerta de Santa Engracia y sus alrededores: "Intramuros de la puerta de la Independencia y á entreambos costados de la misma existían dos solares que miden en totalidad más de 20 áreas obstruidas por escombros e inmundicias, que afectaban mucho aquél sitio tan público y concurrido".

La memoria culmina con las "mejoras en proyecto", un apartado en el que el ingeniero manifiesta la necesidad de "un jardín que sirva de paseo público á semejanza de los que poseen otras ciudades, como centros de concurrencia, con especialidad en los días festivos". En este sentido dice que el salón de Pignatelli o de Santa Engracia - paseo de la Independencia- cumple estas funciones de desahogo y esparcimiento, pero "carece del atractivo y hermosura que indudablemente tendría si su plazoleta o calles laterales estuviesen rodeadas con anchos cuadros de flores y plantas de adorno".

Los profesionales de la naturaleza fueron los primeros en constatar la pobreza de la jardinería y la escasez de lugares de recreo de la ciudad. El testigo de sus peticiones fue recogido por los concejales más progresistas que encontraron en la margen derecha de Zaragoza un espacio por explotar con la intencionalidad de transformarlo en un nuevo espacio burgués, de ahí que se precisaran infraestructuras y espacios acorde a su nueva identidad.

El eje vertebral de esta zona se encontraba en el floreciente paseo de Torrero que conectaba el centro con la zona Sur hasta las aguas del Canal Imperial. En el año 1886 comienzan las obras que debían de reformar y aderezar el paseo y en relación a ello, el concejal Gimeno F. Vizarra presenta una moción en la que insta a la creación de un verdadero lugar para el esparcimiento de la población zaragozana: ${ }^{3}$

"El paseo de la calle de la Independencia, único que existe en la población, no tiene ya suficiente espacio para que los concurrentes al mismo puedan ni aun respirar en las condiciones favorables que la higiene exige para estos sitios de esparcimiento; que se estaba en el caso creando uno nuevo, dando condiciones de tal, al que malamente se titula paseo de Torrero ó á la playa del Canal; que al primero convertido hoy en un túnel, debía de reformarse y sustituir con verjas las tapias de las casas de campo que lo forman, iluminándolo convenientemente; que en cuanto a la playa de Torrero podía prescindirse ó tratarse con los ramos de Guerra y Fomento, á cuyo cargo parecía que se hallaba en la actualidad, viendo la forma de que alguna manera disfrute el público de aquellos terrenos para paseo"

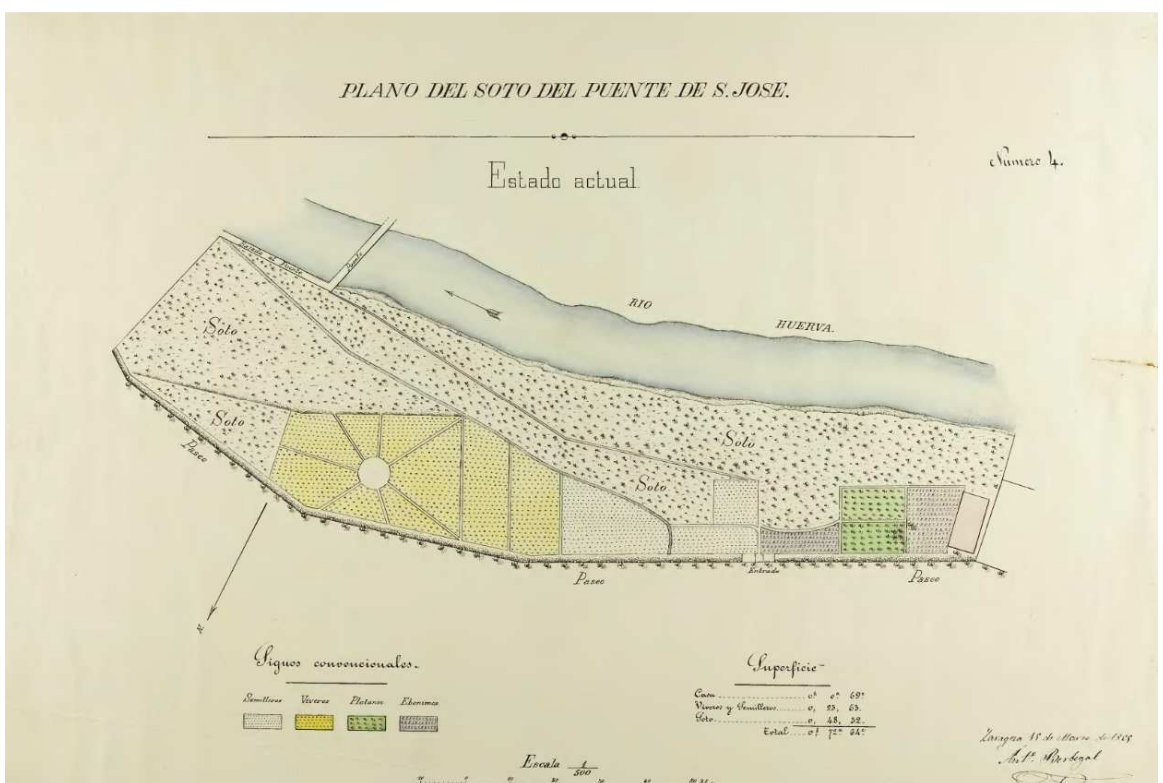

Soto del puente de San José "Memoria sobre el ramo de arbolados y jardines de Zaragoza". (Antonio Berbegal y Celestino, 1862). Fuente: Archivo Municipal de Zaragoza.

\footnotetext{
${ }^{2}$ A.M.Z. Caja 1.420, exp. 454/1865.

${ }^{3}$ Actas Municipales, 16 de julio de 1885 , f. $23 / \mathrm{v}$ y f. $24 /$ r.
} 
Esta voluntad es recuperada en marzo de 1887 por la moción presentada por el alcalde Sainz de Varanda que resume la situación con estas palabras: ${ }^{4}$

\begin{abstract}
"El grande movimiento en la vida moral y material de Zaragoza de pocos años acá, ha hecho pensar al Municipio y al vecindario todo en los medios de verificar el ensanche de la población (...) al propio tiempo de dotarla de algunos paseos cómodos y espaciosos, cuya falta se siente por aquella circunstancia. La glorieta de Pignatelli que servía como punto de solaz y esparcimiento, ha quedado reducida en la actualidad a una pequeña elipse formada entre una serie de mayores y menores edificios y sin espacio capaz para el numeroso público que a diario concurre á dicho punto (...) De aquí la necesidad inmediata del pensamiento para preparar y disponer un punto a donde pueda ir el público a esparcir los ánimos sin la aglomeración que se observa en los sitios hoy reducidos por las edificaciones y ninguno para tal objeto como los que afluyen a Torrero, únicos que quedan en buenas condiciones y el más frecuentado por todas las clases sociales de la ciudad. A esta indispensable mejora, relacionada también con otra sobre las acopias para pavimentos podría dársele formas tomando algunos terrenos que existen entre el paseo de las Acerolas y el camino de Torrero desde Cuellar, hasta frente la iglesia y jardines de aquel punto. Estos terrenos que ha pocos años pudieron adquirirse por muy poco precio costarán mayores sumas cuanto más se dilata la realización de la indicada mejora, cual ha acontecido con el campo llamado de Lezcano"
\end{abstract}

En este mismo año, Gimeno Vizarra da un paso adelante a la iniciativa cuando propone la construcción de un parque o una zona ajardinada junto al paseo de Torrero, a lo que la Sección $2^{\circ}$ del Ayuntamiento emite un informe en el que declara la idea de llevar a cabo un intervención completa y por consiguiente, construir un espacio de solaz "especialmente para niños, donde no se moleste a los transeúntes y haya cierto horizonte del que se va careciendo por las muchas edificaciones llevadas a cabo en las afueras". Así pues, las mejoras se centraron en el paseo de Torrero, al mismo tiempo que se iba conformando como una zona residencial, propia de las clases más elevadas de la población, y seguía en parte los dictados de la moda al uso, basada en el establecimiento de la residencia en el límite de la zona urbana (García Lasaosa, 1979: 135). La remodelación de este punto concreto de la ciudad culminaría con la redacción del proyecto del parque Pignatelli en el año 1892 y su paulatina conformación.

En 1900 el Guarda Mayor de Arboledas y Jardinero Municipal, Francisco Valero se trasladó a París para asistir a la Exposición Universal bajo petición de la Sección 50 del Ayuntamiento con la finalidad de recopilar información valiosa sobre las zonas verdes de la capital francesa para trasladarla y aplicarla en Zaragoza.

Tras permanecer veintidós días en París, el jardinero municipal plasmó sus observaciones en una memoria presentada el 30 de enero de $1901 .{ }^{5}$ Este escrito nos ayuda a conocer la opinión de un trabajador municipal directa vinculado a la formación y mantenimiento de la jardinería. Aunque la memoria es un continuo ataque a la desatención sobre los espacios verdes y la falta de recursos materiales y económicos que impedían desempeñar correctamente las labores de jardinería zaragozanas.

En la primera parte de la memoria dedicada al arbolado establece una comparación entre el arbolado existente en París y Zaragoza y los diferentes sistemas de actuación como el riego o la poda. La situación de los paseos zaragozanos, bajo el juicio de Valero, era deplorable, ya que únicamente el paseo de la Independencia contaba con riego a partir de un sistema de alcantarillado, mientras que el resto a causa de la creciente urbanización y el excesivo movimiento de vehículos no podían recibir el agua necesaria por el estrechamiento de las cunetas. Otro de los problemas que señala es la introducción de las tuberías de gas en lugares como la "Ronda de la Ciudad", el paseo de la Lealtad y el de María Agustín, pero sin duda el mayor causante de la desaparición del arbolado era la urbanización, "no pasara muchos años sin que podamos decir que, el magnífico arbolado de Zaragoza, vive muriendo". Esta era la realidad que estaban sufriendo los principales paseos zaragozanos desde que fueron escogidos por la burguesía para asentar sus residencias, esencialmente por todos valores asociados de embellecimiento natural y de recreación pública. En relación a los jardines de Zaragoza afirma que "son pocos y pequeños".

La gestación de espacios verdes estaba incardinada a la limitación técnica y de infraestructuras y sobre todo a la escasez de agua que fue uno de los problemas crónicos con los que contó el arbolado a lo largo del siglo XIX (Gómez, 2003: 22). A ello se añaden tres factores determinantes: la imposibilidad de reponer las espacies vegetales, un problema que en Zaragoza aumentaba debido a una atmósfera candente en verano que requería la renovación de plantas anualmente; la construcción incontrolada que colmataba el interior de la ciudad a merced de los intereses económicos; las precariedad de las arcas municipales.

\footnotetext{
${ }^{4}$ A.M.Z. Caja 1454, exp. 193/1888, Sobre el rebaje y arreglo del camino y paseos de Torrero.

${ }^{5}$ A.M.Z. Caja 935, exp. 1062/1900.
} 
En suma, la falta de recursos y la creciente urbanización fueron claves para el desarrollo de los espacios verdes. Este último aspecto fue causa y a la vez consecuencia de la integración de la naturaleza en la ciudad a lo largo del siglo XIX, pues como dejan constancia los contemporáneos, el aumento del suelo construido alejaba a los ciudadanos de un entorno natural y ante ello, sus respuestas se encaminaron hacia la ensoñación bucólica de un espacio higiénico que constituyese un marco de relaciones sociales.

\section{UN PARQUE URBANO PARA ZARAGOZA: EL PARQUE PIGNATELLI, UN CONFLICTO DE INTERESES.}

\section{1. Un parque para la ciudad: el proyecto del Parque Pignatelli}

Zaragoza inicia la segunda mitad del siglo XIX desbordada de sus límites tradicionales en algunos puntos a causa del crecimiento demográfico, el desarrollo industrial y la incorporación del tranvía (1885) que conectaba la ciudad con las afueras. Desde el punto de vista social, según María Rosa Jiménez (1979: 289), las clases medias "formarán una oligarquía ciudadana que absorberá los cargos directos de la ciudad" cuyas decisiones provocarán cambios en el paisaje urbano modelado a favor de los intereses particulares. Haciendo así de la esfera pública un espacio representativo del colectivo acomodado.

Los ejes de expansión del ensanche finisecular hacia el Sur fueron la formación del paseo de Santa Engracia y la celebración de la Exposición Aragonesa de 1868 en la glorieta de Pignatelli. Como se ha adelantado en los apartados anteriores, la vía de mayor relevancia en esta zona fue el camino de Torrero, actual paseo de Sagasta.

La prolongación del camino de Torrero se conocía con el nombre de Subida de Cuellar, un tramo que había sido reformado por suscripción de los propietarios del entorno. También en el entorno se localizaban los primeros depósitos de agua que abastecieron de agua a la ciudad (1876) y un terreno ocupado por torres (casas asociadas al trabajo agrícola), cuyos campos de cultivo formaban una superficie triangular que recibía el nombre de Graveras de Cuellar. Este espacio estaba flanqueado por dos vías, el camino o Subida de Cuellar y el camino de las Acerolas.

El arquitecto municipal, Ricardo Magdalena, esbozó sus aspiraciones sobre este terreno y oficialmente presentó al Ayuntamiento el proyecto "de jardines a la terminación de Torrero" el día 29 de febrero de 1892, acompañado de la memoria y del plano de lo que sería el parque Pignatelli (ver apéndice). Un proyecto que suponía la aplicación de los nuevos criterios de planificación urbana, introduciendo un espacio verde que no solo constituyera un lugar beneficioso para la salud pública sino también un motor de prestigio para la capital aragonesa.

Este espacio ajardinado hizo realidad las sucesivas propuestas de embellecimiento de Torrero impulsadas originalmente por la mente liberal de Sainz de Varanda. Además de ello, se abordan otras cuestiones planteadas con anterioridad como la inexistencia de un espacio de recreo en la zona de ensanche de Zaragoza, ya que como explica Magdalena, prácticamente la plaza de Aragón y el paseo de la Independencia se hallaban edificados en su totalidad, por consiguiente: "han perdido el carácter de paseo que antes tenían". La masiva urbanización de estos espacios urbanos habían situado en el punto de mira el paseo de Torrero, el cuál tiempo atrás se había embellecido con la plantación de árboles, sin embargo, su prolongación hasta el Canal, a juicio del arquitecto debía de completarse con un amplio lugar de esparcimiento y descanso.

En el proyecto plantea la ocupación de la totalidad del espacio triangular, bajo la expropiación de todas las fincas, siguiendo un diseño cercano a la tendencia paisajista, muy adecuada para el desnivel de la gravera. El plano muestra una composición formal a partir de dos ejes que constituirían dos plazas, una en forma de herradura de 55 metros y la otra de forma casi elíptica de 60 metros. Mientras que el resto de terreno es surcado por caminos y paseadores ondulantes que delimitan los parterres, una libertad compositiva que contrasta con la gran regularidad de la forma triangular de las graveras. Desde el punto de vista formal, escoge la vertiente "naturalista", utilizada paralelamente en otras ciudades españolas como en el jardín del Campo del Moro de Madrid, en el que, aunque no es semejante en extensión y preeminencia al de Zaragoza, su jardinero Ramón Oliva optó por un entramado similar basado en líneas curvas y pequeñas plazas ovales. Estos trazados "ingenuos" nos recuerdan también a los planeados por el paisajista Barillet Deschamps (1824-1873), jefe jardinero de París o a los del arquitecto paisajista estadounidense Frederick Law Olmsted (1822-1903). Estas relaciones formales no son de extrañar teniendo en cuenta que pocos años atrás, París, se había engalanado con un magnífico programa de parques y jardines en estilo paisajista, gracias a la iniciativa del emperador Napoleón III y el prefecto de la ciudad, Georges-Eugène 
Haussmann que delegaron la dirección de esta propuesta al ingeniero y paisajista Jean-Charles-Adolphe Alphand. La actuación más admirable de esta intervención fue la transformación del Bois de Boulogne (1852-1860), de la cual, según Francesco Fariello (2004:271): "surgió el parque más hermoso que se creó en Europa en aquella época".

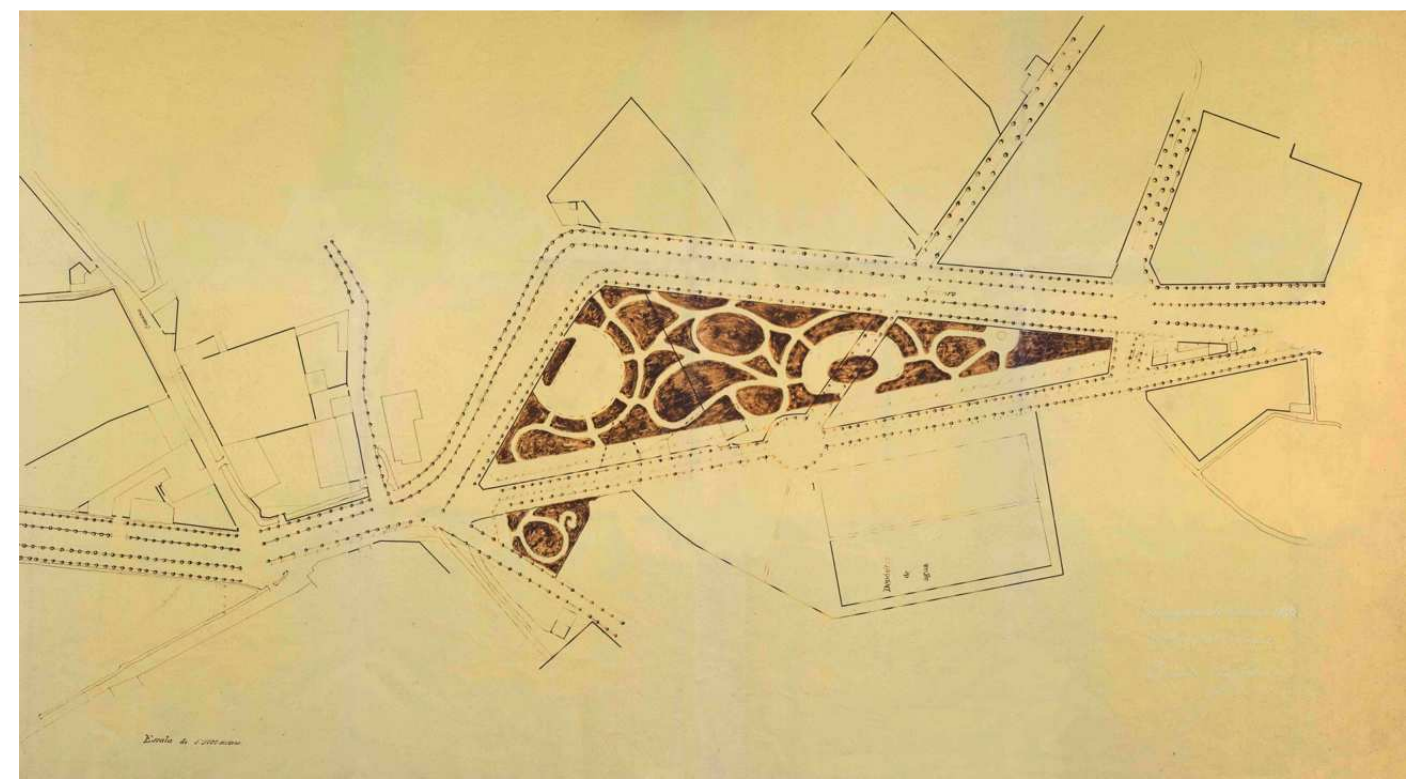

Proyecto "de jardines a la terminación de Torrero" (Ricardo Magdalena, 1892). Fuente: Archivo Municipal de Zaragoza.

El estilo elegido para el diseño del parque Pignatelli y las humildes pretensiones que giraban en torno a él, configuraban un proyecto que por su sencillez no mostraba inconveniente alguno a grandes rasgos, sin embargo, pronto se tornó en conflicto para el Ayuntamiento por las múltiples barreras a las que tuvo que hacer frente, en las que se aunaron los intereses económicos por parte de los propietarios del terreno y del entorno y también, la lucha social de los vecinos por hacer realidad la construcción de un parque para la ciudad.

\section{2. El papel económico y social en torno al parque Pignatelli}

La construcción de nuevos espacios ajardinados fue laboriosa para el Ayuntamiento por la dificultad de disponer de suelo, entre otras cosas, a causa de la existencia de propiedades particulares en el anillo que envolvía el centro urbano, un hecho que encareció las actuaciones del Municipio (Gómez, 2003). En el Sur zaragozano, el arquitecto municipal Ricardo Magdalena ya había advertido en el año 1887 mediante un escrito lo peligroso de que dicha zona comenzara a edificarse sin un plan concebido puesto que supondría en un futuro el gastar fuertes sumas de dinero para la expropiación. Para que esto no ocurriera, aconseja conceder al paseo de Torrero el tratamiento legal de una "vía urbana", lo que exigía la servidumbre de aquellas fincas y edificios que se vieran afectados por la nueva alineación mediante la paulatina expropiación de las mismos. Asimismo, propone un proyecto para prolongar el paseo de Torrero con una nueva vía que corriera paralela a la subida de Cuellar, denominada camino de las Acerolas, quedando en el centro de ambas la gravera de Cuellar, terreno destinado a la construcción de un parque o jardines. Ante la aprobación del mismo, se interpusieron dos demandas de los dos propietarios que poseían sus fincas en las graveras, Joaquín Martón y Pablo Buil ya que esta nueva alineación afectaba a sus fincas.

El primero, abogado y político de gran relevancia en la vida ciudadana, pronto llegó a un acuerdo con el Concejo, posicionándose como partidario de las reformas, de hecho, en 1886, había permitido extraer grava para la realización del primer tramo del paseo de Torrero, por lo que aceptó seguir el procedimiento de expropiación. En 1888 es expropiada la totalidad de su finca de $8930 \mathrm{~m}^{2}$ con la finalidad inicial, de cubrir las necesidades municipales, la extracción de grava y de dar trabajo a los jornaleros en invierno. Los intereses del Ayuntamiento iban más allá de remediar la falta de trabajo en la población durante los meses invernales, tenían presente la revitalización y la notoriedad que estaba viviendo esta zona, por lo que una vez extraído todo el material el valor del solar aumentaría. 
A diferencia de este propietario, Pablo Buil, notario de la Curia eclesiástica y abogado, exigió que se modificara el plano en favor de su finca, pero sus reclamaciones fueron desechadas para cumplir la Real Orden del 12 de marzo de 1878, en la que se establecía la obligatoriedad de servidumbre de los propietarios a favor de las mejoras de la ciudad. Sin embargo, la negativa por parte de Buil se debía a la importancia que había adquirido su torre de verano, en donde recibía a sus amistades para reunirse en tertulia. La expropiación de su finca se prolongó hasta 1898, como se explicará a continuación. También se expropió una fábrica de ladrillos de la sociedad "Hernández, Lagrava y García" que ocupaba el vértice del triángulo de una extensión de $5070 \mathrm{~m}^{3}$, una parte del terreno se destinó para el establecimiento de un escuela pública.

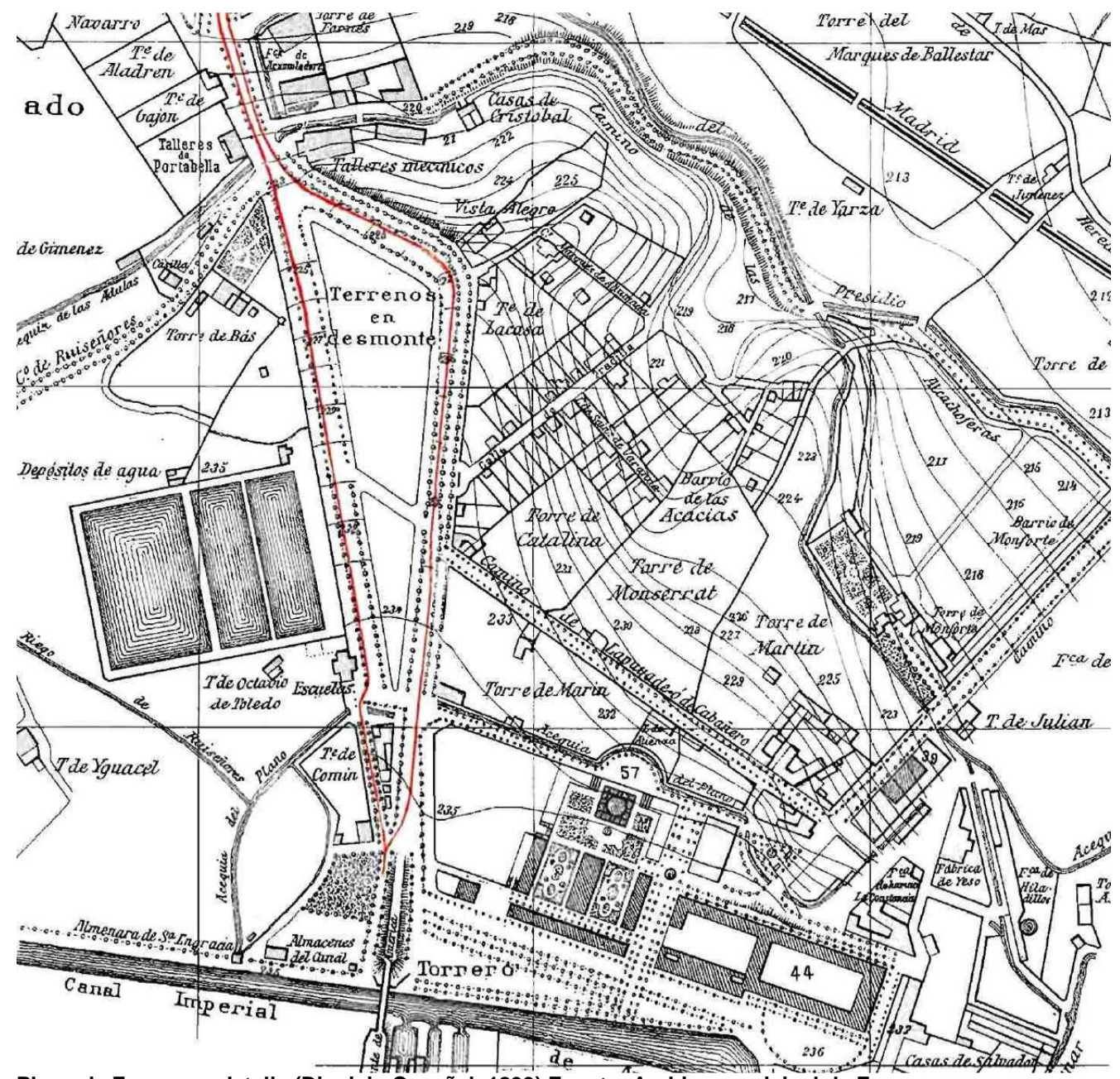

Plano de Zaragoza, detalle (Dionisio Casañal, 1899) Fuente: Archivo municipal de Zaragoza

Los trabajos en las fincas para el desmonte de las graveras de Torrero había comenzado para la extracción de materiales y únicamente quedaba en pie la torre de Pablo Buil. Pese a la imposición de expropiación para ajustarse al proyecto que se había concebido en el paseo de Torrero, en julio de 1890, Buil solicitó licencia para llevar a cabo reformas de ornato en su torre. Sin embargo, la petición es denegada por el arquitecto municipal, de hecho, plantea que lo más conveniente sería expropiar la totalidad de la finca porque existía la idea de realizar unos jardines o un parque de recreo. En noviembre del mismo año, Buil se reunió con el alcalde para expresar sus deseos y aspiraciones en relación a la torre y mostró su disconformidad en cuanto a la cesión voluntaria de la finca, por la cuál exigía una indemnización desorbitada.

Ante la imposibilidad de llegar a un convenio de compra con el propietario, Ricardo Magdalena elaboró detalladamente sus ideales sobre el terreno de la gravera y de manera oficial ante el Ayuntamiento presentó el "Proyecto de jardines a la terminación de Torrero" en 1892. 
Para poder llevar de manera legal la adquisición de la finca de Pablo Buil, la Sección $2^{\circ}$ propone tratar este proyecto de "utilidad pública" y de esta manera aplicar la ley del 10 de enero de 1879 sobre la expropiación forzosa. Con la aprobación del gobernador civil, Francisco Fernández de Navarrate, se publicó la decisión del Ayuntamiento en el Boletín Oficial de la Provincia de Zaragoza (BOPZ) el día 20 de abril de 1892, a la que dio respuesta el único afectado. Buil alegó que era un proyecto "caprichoso, estético e inútil" y se justificó a partir de los siguientes razonamientos jurídicos, económicos y sociales:

- Olvido por parte del Ayuntamiento del acuerdo tomado en cuando al ensanche y urbanización del paseo de Torrero de 1887, cuya alineación, aprobada en 1888, afectaba unos metros a su finca. Ahora con el proyecto que se planteaba se excedía la línea acordada porque tomaba por completo toda la finca, por lo que no podía considerarse "ni legal, ni jurídica, ni gramatical, ni obra de utilidad pública".

- Que ni en la Ley sobre la utilidad pública, ni en la de expropiación forzosa se hace referencia a la expropiación para la construcción de un jardín, una obra con una finalidad más estética que funcional. Para Buil esta obra demuestra que su finalidad no es social sino de "atacar indirectamente a un derecho individual".

- El gran desembolso económico que supondría la creación de jardines, puesto que en el proyecto ni se incluyen las expropiaciones ni el desmonte de la gravera, y pone en duda que el valor del desmonte esté cubierto con la adquisición de materiales para obras públicas. ${ }^{6}$

Estos razonamientos darían lugar a la respuesta de la Sección $2^{\circ}$, declaran: "la actividad del capital no consiste en solo producir dinero, sino en traducirse en utilidades de distintas clases, no porque a veces no se puedan apreciar con números, dejan de significar un producto real y positivo", además manifiestan como tratan de evitar el gasto que supondrían los terrenos de la subida de Cuellar en el futuro. El Ayuntamiento acudió al gobernador civil para la aprobación definitiva del proyecto y finalmente el 12 de junio de 1893 se declaraba el "Proyecto de jardines a la terminación del paseo de Torrero" de utilidad pública. Entre los motivos que se expusieron para ello fue la necesidad de construir un parque en una población en la que no existía un pequeño espacio para niños y personas, acorde a las exigencias de higiene y salubridad.

Ante el acuerdo, Buil elevó la instancia al Supremo, al Ministerio de Fomento, suplicando que todo lo anterior se declarara nulo, acogiéndose al derecho de propiedad. La resistencia del propietario se debía principalmente al aumento de valor que su torre iría adquiriendo con el trascurso del tiempo y de la necesidad por parte del Ayuntamiento de obtener la parte restante de su finca a un precio más elevado. El director General de Obras Públicas, remitió la Real Orden del 5 de noviembre de $1893^{7}$ en la que manifestaba que la prolongación del paseo de Torrero y la construcción de un parque respondían a dos proyectos y leyes distintas, en el primero se aplicaba la servidumbre, mientras que en el segundo la expropiación forzosa. Asimismo, la Real Orden declaraba que el ensanche del paseo de Torrero era de utilidad pública, de tal manera que había que expropiar la parte de Buil acordada relativa a este proyecto. Mientras, que la declaración del utilidad pública para los jardines fue revocada por no estar debidamente justificada. Esta abolición se debe a que la finalidad era eminentemente de recreo y de carácter estético. Concluye con la obligación a la Corporación Municipal de llevar a cabo de manera inmediata la expropiación del terreno necesario de la finca de Pablo Buil para el ensanche del paseo de Torrero, ya denominado de Sagasta.

La torre de Buil se expropió en los metros acordados en el primitivo proyecto del camino de Torrero, tras una década de resistencia por parte del propietario, y siguiendo los consejos de sus allegados, el 31 de Octubre de 1898 se firma la escritura de venta a favor del Ayuntamiento por el precio de 75.000 pesetas.

La visión de ciudad compacta mostrada por Zaragoza hasta el siglo XIX quedó fragmentada a causa del crecimiento incontrolado del extrarradio, que comenzó a ser ocupado por barriadas obreras habitadas por aquellos que habían emigrado del campo a la ciudad. El paisaje fue transformándose con el tiempo, cambiando las torres por industrias y chalets, aumentando la proporción de suelo construido y creando una imagen de la ciudad y de sus afueras cada vez más cosmopolita. Esta cuestión preocupaba a las autoridades de la ciudad que seguían mostrando sus esperanzas en la construcción de un parque urbano para la población zaragozana.

Tras un breve paréntesis en los trabajos en las gravera, en 1902, el concejal Ojeda presenta una moción en la que insta a retomar el desmonte y construir el deseado parque. A esta reivindicación se sumaron los

\footnotetext{
${ }^{6}$ A.M.Z. Caja 955, exp. 55/1905, ff. 21-28.

${ }^{7}$ A.M.Z. Caja 567, exp. 339/1898, ff. 57-75.
} 
vecinos de las fincas contiguas para condenar el estado de las graveras tras la adquisición de la torre de Buil, los cuáles apoyarán incondicionalmente el proyecto del parque y harán de la prensa local, como veremos a continuación, su máximo aliado. Un vecino anónimo de la ciudad de Zaragoza en una carta dirigida al alcalde Amado Laguna publicada en el Diario de Avisos de 9 de mayo de 1903, exponía lo siguiente:

"Treinta años hace que el excelentísimo Ayuntamiento trató del desmonte del cabezo ó promontorio que cual muralla de la China, separaba el paseo de Torrero de las risueñas playas del Canal Imperial y seguimos hoy como hace treinta años, es decir bastante peor (...) Nosotros, los de casa, ya nos habíamos habituado á ver cosa tan fea, ¿pero que idea se llevarán los extraños á quienes como número obligado convidamos para que contemplen los alegres paisajes de Torrero? Tener condenado uno de los sitios más públicos de la afueras a eterna y antiética gravera con sus socavones y escondrijos gratos solo para gentes maleantes, es el colmo de la incuria y el abandono. (...) Esto, Sr. Laguna, no puede ni debe continuar así y si V.E. con decisión firme no completa la obra iniciada por el malogrado patricio D. Francisco Cantín, antes de que la inestabilidad de los cargos públicos tan corriente en esta nación, lo obligue á dejar la casa, el entusiasta aplauso que le guardamos para cuando aquel indecoroso lugar lo haya V.E convertido en jardín frondoso, habrá de trobarse en acerba y honda censura" (Diario de Avisos, 1903 a: 1).

Las intencionalidades de los vecinos se debatieron en una primera reunión celebrada el 15 de junio de 1903, presidida por el alcalde Laguna, la Sección 5o y varios propietarios de la zona. En ella, los propietarios se comprometieron a reunir dinero para cubrir los gastos de la mejora y se acordó que el arquitecto municipal formara de nuevo un presupuesto relativo a las explanaciones. "Este procedimiento no era la primera vez que se ponía en práctica, ya que en varias ocasiones se ha comprobado como los vecinos de determinadas calles, como por ejemplo la de Alfonso, colaboraban económicamente con el Municipio a fin de sufragar el coste producido por la instalación de diferentes servicios como los de alumbrado, pavimentación, etc. Con ello, conseguían una rápida ejecución de sus mejoras" (García Lasaosa, 1979: 195).

El importe establecido por el arquitecto fue de 2.500 pesetas para el desmonte de la gravera y a ello había que sumar la conformación de jardines. Para hacer frente a los gastos, los propietarios anunciaron la creación de una sociedad, llamada "La Actividad" y como representantes de la misma se nombró a José Salas y Melchor Camón. En la reunión se trató el asunto con una perspectiva de futuro y quedó de manifiesto los intereses económicos que despertaba el parque, puesto que beneficiaría a las fincas que flaquearían el terreno cuyos solares se revalorizarían. Las expectativas generadas sobre la creación de un parque en la subida de Cuellar contribuyó a revalorizar del suelo de los terrenos adyacentes.

El Alcalde determinó que debido al estado deplorable de las arcas municipales debía de ser esta sociedad la encargada de hacer frente a los gastos económicos de la reforma.

Entre los integrantes de la sociedad, destacar a Julio Juncosa, Emilio Ostalé, Juan Salinas, José Gascon, Damian Escudero o Mariano Aladrén. Todos ellos pertenecientes a la burguesía local zaragozana y vinculados de una forma u otra al gobierno local de Zaragoza.

También la vecindad propuso un diseño específico para el parque, tal y como se recoge en un ensayo publicado en el Diario de Avisos el 15 de julio de 1903:

"Dejando a un lado los mil modelos que podríamos copiar del extranjero y que V.E. conoce perfectamente, también dentro de casa como quien dice, tenemos ejemplos á que referirnos, pues en poblaciones donde el terreno es llano por su natural topografía, hemos visto que a costa de enormes gastos de trasporte y terraplenado, se hacen montículos artificiales para sustituir la monótona planicie por la placida variedad de una superficie caprichosamente ondulada y sin embargo hay quien opina que se debe gastar mucho dinero en hacerlos desaparecer para ajustarse a la rasante uniforme, es ya el colmo de los inconcebible (...) Como el diámetro de la gravera afecta la forma de un triángulo de mucha mayor longitud que anchura, la pendiente en sentido longitudinal resulta relativamente suave, siendo algo más fuerte en el sentido transversal. Ambos declives determinan dos planos de inclinación distinta y el buen efecto de las ondulaciones y pequeñas colinas más o menos elevadas dentro de estos dos planos horadados por la línea actual del tranvía, dependerá del mayor gusto o acierto de la persona encargada del replanteo de los jardines" (Diario de Avisos 1903 b: 1).

El 2 de Septiembre se volvieron a reunir con el dinero recaudado: 8.000 pesetas por parte de los vecinos y 5.000 pesetas subvencionadas por el Ayuntamiento, para el pago de los gastos del desmonte de las graveras de una superficie de $13.000 \mathrm{~m}^{2}$ al contratista de las obras, y se fijó su terminación en veinte días, antes de la festividad del Pilar. El propósito era comenzar con las labores de jardinería tras la terminación de las fiestas, donde pasaría a intervenir la Sección de Agricultura bajo la dirección técnica del Arquitecto 
Municipal. ${ }^{8}$ La junta directiva nombrada por los vecinos agradecieron al alcalde la atención recibida para este gran progreso:

"La realización de una mejora tan necesaria y conveniente como lo es el dotar a la ciudad de un parque o jardín donde especialmente los niños sin riesgos de ningún género puedan proporcionar aire sano a sus pulmones, alegría a su espíritu infantil y fuerza a sus delicados músculos para que siendo fuertes físicamente, puedan serlo también en el orden intelectual y sean igualmente un día la grata esperanza de la anhelada Patria Grande".

El 10 de octubre de 1903 el Diario de Avisos confirmaba la terminación de la explanación de las graveras. Así pues, poco a poco, la antigua gravera se fue convirtiendo en una extensa zona ajardinada que mucho se alejaba del proyecto originario de 1892.

Resultaba evidente que la zona de Torrero era la preferida por los zaragozanos en los días festivos, tal es así que el 20 de Noviembre de 1903 se aprueba la construcción de un parque mesocrático en el Cabezo de Buenavista (Monte de Torrero) de 21 hectáreas, actual parque José Antonio Labordeta. Esta nueva iniciativa situó la construcción del Pignatelli en un segundo plano, aunque todavía quedaba una última pretensión en torno a él. El 5 de febrero de 1904, el concejal Alfonso en sesión ordinaria expuso el gasto inmenso que supondría la construcción definitiva de los jardines de la subida de Cuellar, así como su conservación, y en su lugar, entiende como una solución acertada la parcelación del terreno y su urbanización con hoteles y jardines a la inglesa. Calcula una inversión de unas 200.000 pesetas con un beneficio de entre 35.000 a 40.000 pesetas, una pretensión del concejal que se debía al ya consolidado carácter burgués que había adquirido la zona. La moción fue rechazada por varias razones, como combatió el concejal Alfredo Ojeda: "defraudaría las esperanzas de todos y tan solo conseguiría que los pudientes hicieran allí sus chalets".

A partir de este momento, el espacio del parque Pignatelli se fue consolidando poco a poco tal y como refleja la documentación de la época, aunque en 1905 un artículo del Heraldo de Aragón ponía al corriente la verdadera situación de las afueras de la ciudad, concretamente de la parte Sur con motivo de la llegada del verano a Zaragoza:

“¿Quién se atreverá a salir de su casa en nuestra ciudad, durante esas horas terribles, si quehaceres urgentísimos no le apremian? No hay parques interiores que hagan de oficios de pulmones, ni arboles corpulentos que den suave y fresca sombra, ni nada en fin halagador y confortable, sino un sol despiadado que funde las aceras y arranca chispas de los adoquines" (Heraldo de Aragón, 1905: 1)

A pesar de ello, las obras para la finalización del parque Pignatelli se prolongaron hasta los años treinta del siglo XX, aunque sus remodelaciones se sucedieron hasta finales del siglo para adecuar sus infraestructuras a las nuevas necesidades de la sociedad. Al mismo tiempo, la ciudad de Zaragoza se dotó de más de una treintena de parque urbanos y otras tipologías de espacios verdes al compás del surgimiento de nuevos barrios y gracias a la asimilación de nuevas concepciones en torno a la inserción de la naturaleza en el ecosistema urbano.

La historia del parque Pignatelli es inconclusa, en la actualidad se han esbozado nuevos ideales en torno al parque más antiguo de la ciudad. El espíritu del nuevo proyecto sigue la línea de su historia, ya que el Ayuntamiento desde los últimos años ha desarrollado una serie de actividades participativas basadas en las sugerencias expresadas por el vecindario relacionadas con las necesidades del entorno. De la nueva intervención en el parque se espera su ampliación por el terreno anexo de los depósitos de agua, con la incorporación de equipamientos deportivos, culturales y cívicos, pero también la edificación junto a él de una nueva zona residencial selecta que contribuirá a la financiación del proyecto (Heraldo de Aragón, 2016). Todavía son bosquejos de un futuro que se prevé no muy lejano.

\footnotetext{
${ }^{8}$ Actas Municipales, 4-9-1903, f. 292.

${ }^{9}$ Actas Municipales, 5-2-1904, ff. 34-37.
} 


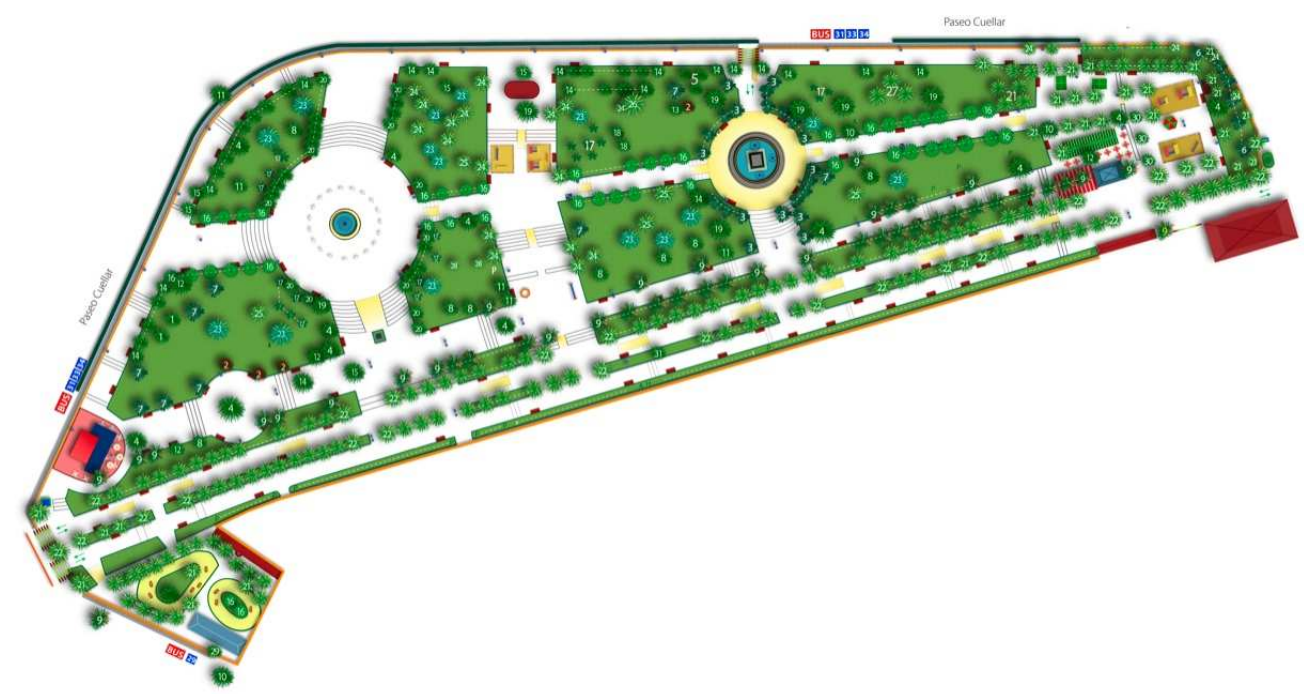

Parque Pignatelli en la actualidad. Fuente: $\underline{w w w . p a r q u e s z a r a g o z a . e s}$

\section{REFLEXIONES FINALES}

En términos generales, los trabajos de jardinería puestos en marcha por el municipio zaragozano a finales del siglo XIX se pueden calificar de puntuales y limitados a la incorporación de las formas tradicionales utilizadas en la proyección de la naturaleza, la plaza ajardinada y el paseo arbolado. Esta realidad, definida por la inexistencia de nuevas tipologías de espacios verdes y de un imponente trazado urbano surcado por vegetación, evidencia la desconexión en materia de actuación urbanística entre la capital aragonesa y las propuestas que se estaban implantando en el ámbito nacional e internacional. En este caso, los arquitectos, los profesionales de la naturaleza locales y las mentes más progresistas de la ciudad conformaron el motor intelectual que acabó instaurando las aspiraciones paisajistas, iniciando el cambio y el despertar del urbanismo zaragozano. En relación a ello, no hay que olvidar que la constitución del parque Pignatelli estuvo envuelta en un debate público (testimoniado en los plenos municipales y en la prensa zaragozana), en la que el hombre se sublevó ante la desnaturalización que había sufrido la ciudad a lo largo del XIX, al mismo tiempo que sus exigencias eran propias de su tiempo, de los nuevos privilegios nacidos en torno al negocio del suelo.

El parque Pignatelli nació como expresión de la necesidad colectiva y se desarrolló como un laboratorio de pruebas sin precedentes, de hecho, del primer diseño presentado por Ricardo Magdalena a lo que ha llegado a ser en nuestros días hay un abismo y en parte se debe al sometimiento de su constitución inicial a la opinión ciudadana y por supuesto, a la aplicación de las corrientes paisajísticas y al cambio de los usos de la población. También, como primer parque proyectado en la ciudad, despertó una nueva conciencia pública y privada que situó el verde urbano como una fuerza económica eficaz y rentable que lejos de ser ignorada, supuso el despegue de un nuevo espacio como elemento de progreso urbano que dio paso a múltiples propuestas que se acometieron a lo largo del siglo XX.

Por lo tanto, nos hallamos ante un significativo espacio urbano para entender la historia del urbanismo zaragozano que ilustra un arduo proceso determinado por cuatro factores: sociedad, economía, salubridad y estética, aspectos que se conjugaron en un determinado marco urbano y lo llenaron de sentido y vitalidad. A pesar de ser unos factores recurrentes en la proyección de los parques urbanos desde sus orígenes, cada uno de ellos es testimonio de un trasfondo histórico, de la investigación, de la interpretación humana, de la construcción tanto mental como factual que sin duda, contribuyen en el entendimiento de la concepción global de las ciudades. 


\section{BIBLIOGRAFÍA}

CAPEL, H. (2002). La morfología de las ciudades: I. Sociedad, cultural y paisaje urbano. Barcelona: Ediciones Serbal.

FARIELLO, F . (2004). La arquitectura de los jardines: de la Antigüedad al siglo XX. Barcelona: Reverté. GARCía LASAOSA, J. (1978). Desarrollo urbanístico de Zaragoza (1985-1908). Zaragoza: Institución Fernando el Católico.

GÓMEZ, J. (2003). El gobierno de la naturaleza en la ciudad. Ornato y ambientalismo en el Madrid decimonónico. Madrid: Real Academia de la Historia.

JIMÉNEZ, Mo R. (1973). El municipio de Zaragoza durante la regencia de María Cristina de Nápoles: 18331840. Zaragoza: Institución Fernando el Católico.

LÓPEZ, J. J. (1985). Zaragoza a finales del siglo XVIII (1782-1792). Valladolid: Ámbito Ediciones.

MADOZ, P. (1985). Diccionario geográfico-estadístico histórico de Aragón, tomo III: Zaragoza [Edición facsímil 1845-1850). Zaragoza: Diputación General de Aragón.

YESTE, I. (2003). Reforma interior y ensanche en la segundo mitad del siglo XIX en Zaragoza: el plano geométrico. Revista Artigrama (Zaragoza), 19, 427-521.

\section{PRENSA ESCRITA}

(09/05/1905a) De puertas para afuera: carta abierta para nuestro muy querido y respetado alcalde D. Amado Laguna, Diario de Avisos, p. 1.

(15/07/1903b) El parque de Torrero: algunas urgentes indicaciones acerca del mismo que sometemos al recto criterio de nuestro celoso alcalde don Amado Laguna, Diario de Avisos p. 1.

(27/06/1905) El veraneo en Zaragoza: los alrededores de la ciudad, Heraldo de Aragón, p. 1.

(06-10-2016) Un estanque, jardines y 42 viviendas para los antiguos depósitos de Pignatelli, Heraldo de Aragón. [En línea] Disponible en:

http://www.heraldo.es/noticias/aragon/zaragoza-provincia/zaragoza/2016/10/06/un-estanque-jardinesviviendas-para-los-antiguos-depositos-del-pignatelli-1099464-301.html

\section{REFERENCIAS BIBLIOGRÁFICAS}

ASENCIO CERVER, F. (1994). Urbanspaces II: urbanparks, Barcelona: Arco.

DOMĖNEC ROS, J. (2011). Los parques urbanos, algo más que espacio verde. Paisea: revista de paisajismo (Valencia), 19, 107-112.

Borrás Gualís, G; Forcadell Álvarez, C; Yeste Navarro, I. (2009). Zaragoza, 1908-2008: arquitectura y urbanismo. Zaragoza: Demarcación de Zaragoza del Colegio Oficial de Arquitectos de Aragón.

García Hermosilla, C. (1998). Los parques urbanos de Barcelona y Montreal durante el siglo XIX. En CAPEL SÁEZ, H (coord..), Barcelona-Montreal: desarrollo urbano comparado (371-376). Barcelona, Universidad de Barcelona.

YESTE I. (2000). Una aproximación al urbanismo de Félix Navarro. El ensanche de Zaragoza de 1880. Revista Turiaso (Teruel), XV, pp. 175-190.

Yeste NavarRo, I. (2009). Del centenario de los Sitios a la exposición internacional de 2008. En García GuATAS, M, LORENTE, J. P, YESTE, I (eds.). XIII Coloquio de arte aragonés: Zaragoza, 1908-2008 (11-62). Zaragoza: Institución Fernando el Católico, Universidad de Zaragoza, Departamento de Historia del Arte. 


\section{APÉNDICE \\ Proyecto de jardines a la terminación de Torrero. Ricardo Magdalena. Caja 955 exp. 50/1905 ff. 5-8.}

"La creciente importancia que de día en día adquiere el paseo de Torrero obliga al Excm Ayuntamiento á proporcionar comodidades á los paseantes. Terminado casi en su totalidad las edificaciones de la de la Independencia y plaza de Aragón, han perdido el estas el carácter de paseo que antes tenían, y de aquí que el público haya mostrado preferencia por el de Torrero, dotado de buenos árboles y mejorando algunos años en sus condiciones á fin de atender á necesidades que venían sintiéndose.

Ya anteriores Ayuntamientos con tendencias siempre á facilitar comodidades al público, acometieron la empresa de continuar este paseo por el camino de las acerolas, mejora que ha sido muy bien recibida no solo por que satisface exigencias públicas, sino también porque facilita gran cantidad de trabajo á los jornaleros y se obtienen materiales en abundancia para otras obras municipales.

No sería sin embargo la mejora completa si la prolongación mencionada quedase en un profundo desmonte y cerrado por lo dos costados, ni por otra parte es de buen efecto que al finalizar este paseo no haya algún sitio de esparcimiento y descanso.

Comprendiéndolo así el digno ex alcalde Sr. Varanda hizo estudiar á esta oficina un proyecto de extenso parque, cuya realización no propuso á la Corporación Municipal ante el temor de su mucho coste. Mas no por eso debe olvidarse la idea sino que, antes bien, paulatinamente y á medida que los fondos municipales lo permitan es conveniente acometerla. A este fin, á no dudar ha concebido el $\mathrm{Sr}$ Alcalde el pensamiento de dar comienzo á dicho proyecto, estableciendo jardines en los terrenos que indica el plano adjunto, y por ello ha encargado que redacte el oportuno proyecto. Este tiene la ventaja de que las expropiaciones son de poca consideración, puesto que adquirida ya la torre que fue de Don Joaquín Martón, no queda de tanta importancia otro terreno que la torre contigua, puesto que las construcciones más inmediatas al Canal son de escaso valor.

Continuando la obra empezada en la que fue torre del Sr. Martón, debería el resto del terreno rebajarse, dándole la rasante de unión entre el nuevo camino y el antiguo, utilizándose con este operación la grava, piedra y arena resultante, en otras obras y arreglando después el suelo para recibir las plantaciones.

Estas se disponen según indica el plano, dejando en dos sitios distantes otras tantas plazas bastante espaciosas, protegidas por arbolado para obtener sombra y con gran cantidad de bancos para descanso de los paseantes. La más próxima á la Ciudad tiene forma de herradura con un diámetro de 55 metros, y la segunda, de forma casi elíptica cuya eje mayor es de sesenta metros. El resto se halla distribuido en macizos de diferentes formas, separados con paseos de tres metros de latitud, y al final de los jardines se sitúa una pequeña fuente de adorno.

Formando parte del mismo pensamiento, también se proyectan jardines en el pequeño espacio de terreno situado á la derecha del nuevo camino, delante de la posesión del Sr. Blas, con la idea de ampliarla á medida que el Excmo. Ayuntamiento pueda destinar fondos por ese objeto.

Tal es el proyecto que ahora se formula como factible y del que se podrá formar juicio examinando el plano adjunto.

Debo manifestar que el presupuesto aproximado sin contar las expropiaciones cuyo no es fácil averiguar con exactitud será el siguiente:

Jardines 16080 pesetas

Bancos 2960 pesetas

Fuente 1500 pesetas

Total 20540 pesetas

El desmonte tampoco se incluye puesto que los productos compensarán con creces el rebaje del terreno.

Zaragoza 29 de Febrero de 1892

Ricardo Magdalena 